\title{
Mastectomy Scar Boost Results in Low Risk of Locoregional Recurrence in the Setting of Close or Involved Surgical Margins
}

\author{
Laura Johnson, Natalie Lichter, Mamie Hextall, Patricia L. Watkins, Tarek A. Dufan, \\ John M. Watkins
}

c/o Bismarck Cancer Center, School of Medicine, University of North Dakota (Southwest Campus), Bismarck, USA.

Email: john.m.watkins.md@hotmail.com

Received January $6^{\text {th }}, 2014$; revised January $30^{\text {th }}, 2014$; accepted February $6^{\text {th }}, 2014$

Copyright (c) 2014 Laura Johnson et al. This is an open access article distributed under the Creative Commons Attribution License, which permits unrestricted use, distribution, and reproduction in any medium, provided the original work is properly cited. In accordance of the Creative Commons Attribution License all Copyrights (c) 2014 are reserved for SCIRP and the owner of the intellectual property Laura Johnson et al. All Copyright (c) 2014 are guarded by law and by SCIRP as a guardian.

\begin{abstract}
Background: Several Phase III randomized trials have demonstrated improved local control and survival for post-mastectomy radiotherapy in patients with high-risk pathologic features. Close or involved surgical margins were not included as high-risk in these protocols, but have been associated with increased risk of local failure; however, the impact of a boost dose following chestwall radiotherapy in this setting remains to be determined. Methods: Retrospective single-institution outcomes analysis for patients with close or involved surgical margins treated with post-operative radiotherapy is followed by a boost. Results: Between 2003 and 2011, 34 patients were identified for inclusion in the present study. The median chestwall dose was 5040 cGy (range 5000 - 5040) and median boost dose was 1080 cGy (900 - 1620). At a median follow-up of 38.4 months $(10.2$ - 115.6; with $29 \%$ more than 5 years), 28 patients were alive without evidence of recurrence, 3 were alive with recurrent disease ( 1 chestwall), and 3 had died (none with recurrent disease). The 3-year local control, disease-free survival, and overall survivals were $96.9 \%, 93.9 \%$, and $93.1 \%$, respectively. Conclusion: Chestwall radiotherapy plus boost results in low risk of early locoregional recurrence for women with close or involved surgical margin(s) at mastectomy. Further investigation of PMRT with or without boost in this setting is warranted.
\end{abstract}

\section{KEYWORDS}

Breast Neoplasms; Mastectomy; Adjuvant Therapy; Radiotherapy

\section{Introduction}

Randomized trials have demonstrated that post-mastectomy radiation therapy (PMRT) reduces the risk of loco-regional recurrence (LRR) by 50\% - 65\% for women with high-risk features at mastectomy [1-4]. These highrisk pathologic features included invasion of the skin or pectoralis fascia, tumor size $\geq 5 \mathrm{~cm}$, and/or lymph node involvement [2-4]. While surgical margin status was not specifically recorded, analysis of patients with skin and/ or deep fascia invasion (subsets with anticipated high likelihood of margin involvement) demonstrated significant reduction of LRR at 10 years (40\% vs. 7\%) [3,4]. These trials employed standardized regimens (48 - 50 Gy over 4 - 5 weeks via electrons or 37.5 Gy over 3 - 4 weeks via tangents in the European and Canadian protocols, respectively), with mandatory comprehensive nodal irradiation (including both axillary and internal mammary nodal targets) $[2,4]$. No boost was employed in the setting of close or involved surgical margins, and for the overall study populations, the LRR was 5\% - 10\% for irradiated patients.

Retrospective studies of node-negative patients who did not receive PRMT have demonstrated increased risk of LRR in the setting of close or involved margins $(<2$ $\mathrm{mm}$ ) when compared with more widely negative margins [5,6]. While the impact of PMRT in this setting is 
unclear [7,8], evidence from breast preservation studies suggests that a "boost" to the tumor bed decreases LRR beyond that of whole-breast irradiation alone $[9,10]$, and this benefit was preserved with elevated doses in the setting of close or involved surgical margins [11]. In the setting of PMRT, registry-based data suggest improved LRR rates with higher-dose radiotherapy [12,13]; however, the impact of boost doses was implied rather than directly studied. The present investigation seeks to supplement the current literature by defining disease control, patterns of failure, and survival rates among women with close or involved margins who received PMRT with boost.

\section{Methods \& Materials}

This retrospective review was approved by the University of North Dakota, Sanford Health, and St. Alexius Medical Center institutional review boards. Eligible patients were identified from electronic medical records of patients diagnosed with breast cancer between 2003 and 2011, who received PMRT plus boost at the Bismarck Cancer Center. PMRT was employed following chemotherapy (if indicated, at the discretion of the medical oncologist and patient), and was accomplished via megavoltage photons (6 - $10 \mathrm{MV}$ ) via tangents (with or without subsegmentation) or static intensity modulated radiation therapy. Standard fractionation (1.8 - 2 Gy per once-daily fraction) was employed, with chestwall doses of 50 - 50.4 Gy and boost doses of 9 - 16 Gy, at the discretion of the treating physician. The boost volume included the surgical incision plus $2-3 \mathrm{~cm}$ margin uniformly, treated with electrons (multifield if necessary owing to size/convexity of the target, with or without bolus based upon target thickness and electron beam energy employed). Regional lymphatics treated as clinically indicated, generally in the setting of pathologically involved lymph nodes at mastectomty. Patients were required to have had histologically confirmed invasive or in situ breast carcinoma, with close $(\leq 2 \mathrm{~mm})$ or involved/positive margins at mastectomy. This cutoff value was selected based upon previously published data demonstrating increased risk of LRR [5,6].

Study data were collected from existing quality assurance databases and electronic medical records, and included patient demographics, tumor characteristics (including tumor location, histology, tumor grade, hormone receptor status, HER2 status, surgical margin specifics, and pathologic stage), treatment factors (including radiotherapy and systemic therapy specifics), and outcome variables (including patterns of failure and survival). Pre-operative chemotherapy was permitted for inclusion in the present study, with both pre- and post-chemotherapy staging information recorded.
The outcome variables measured in this study included freedom from locoregional failure (FFLF), overall freedom from failure (FFF), and overall survival (OS). All endpoints were measured from the date of initial tissue diagnosis (biopsy), with events recorded at time of first detection.

SPSS 21.0 for Windows (SPSS, Inc., Chicago, IL) was used to analyze demographic and clinical characteristics of patients. The Kaplan-Meier method was employed to describe estimates of disease control and survival.

\section{Results}

Between 2003 and 2011, 34 patients met criteria and were included in the present study. Patient demographics and tumor characteristics are shown in Table 1. Of note, all patients were white and only one patient had a prior history of cancer. No cases of isolated in situ carcinoma were identified; all had invasive carcinoma, and lymphovascular invasion was specifically identified in 23 patients.

Five patients underwent chemotherapy prior to mastectomy; for the remaining 29 patients, the median interval from biopsy to mastectomy was 14 days (range, 0 63). All but one patient underwent surgical nodal staging at the time of mastectomy. The median number of lymph nodes removed was 9 (range, 0 - 34), with 9 patients undergoing sentinel lymph node biopsy only. For 24 patients with involved lymph nodes, the median number involved was 5 (range, 1 - 20), with extranodal extension observed in 18 patients.

Pathologic stage breakdown is demonstrated in Table 2, and detailed treatment characteristics are summarized in Table 3. Of note, 25 patients received radiotherapy to the regional lymphatics in addition to the chestwall (dose range 5000 - $5040 \mathrm{cGy}$ ).

At a median follow-up of 38.4 months (10.2 - 115.6; including $29 \%$ with $>5$ years), 28 patients were alive without evidence of recurrence, 3 were alive with recurrent disease (1 LRR at the chestwall), and 3 had died (none with recurrent disease). As demonstrated in Figure 1, the 3-year FFLF were 96.9\% (95\% C.I.; 95.9\% - 97.9\%), with only one patient having chestwall failure. The overall 3-year FFF was 93.9\% (92.5 - 95.3\%). Three-year OS was $93.1 \%$ (91.5 - 94.7\%).

\section{Discussion}

Following mastectomy, LRR is an unanticipated and concerning site of cancer recurrence. Data suggests that approximately half of patients who experience isolated chestwall failure will subsequently develop distant metastasis within 5 years of LRR, including a 25\% risk for the subset of patients with initial pT1-2N0 disease at 
Table 1. Demographics and tumor characteristics.

\begin{tabular}{|c|c|c|}
\hline Variable & $\begin{array}{l}\text { Median } \\
\text { (Range) }\end{array}$ & N (\%) \\
\hline $\begin{array}{l}\text { Ageat Diagnosis } \\
\text { Median } \\
\text { (Range) } \\
\leq 50 \text { yrs }\end{array}$ & $\begin{array}{l}59 \text { yrs } \\
(27-87)\end{array}$ & $8(24)$ \\
\hline $\begin{array}{l}\text { Method of Detection } \\
\text { Screening Mammogram } \\
\text { Self breast exam } \\
\text { Clinical breast exam }\end{array}$ & & $\begin{array}{l}5(15) \\
28(82) \\
1(3)\end{array}$ \\
\hline $\begin{array}{c}\text { Histology } \\
\text { Invasive Ductal } \\
\text { Invasive Lobular } \\
\text { Mixed Ductal/Lobular }\end{array}$ & & $\begin{array}{l}24(71) \\
9(26) \\
1(3)\end{array}$ \\
\hline $\begin{array}{l}\text { Laterality } \\
\text { Right/Left }\end{array}$ & & $19(56) / 15(44)$ \\
\hline $\begin{array}{c}\text { Estrogen Receptor } \\
\text { Positive } \\
\text { Negative }\end{array}$ & & $\begin{array}{c}26(76) \\
8(24)\end{array}$ \\
\hline $\begin{array}{c}\text { Progesterone Receptor } \\
\text { Positive } \\
\text { Negative }\end{array}$ & & $\begin{array}{l}21(62) \\
13(38)\end{array}$ \\
\hline $\begin{array}{l}\text { HER2 Status }^{\mathbf{a}} \\
\text { Positive } \\
\text { Negative }\end{array}$ & & $\begin{array}{l}10(29) \\
23(68)\end{array}$ \\
\hline $\begin{array}{c}\text { Surgical Margin } \\
\text { Involved/Positive } \\
<1 \mathrm{~mm} \\
1 \mathrm{~mm} \\
1.5 \mathrm{~mm} \\
2 \mathrm{~mm}\end{array}$ & & $\begin{array}{l}18(53) \\
16(47) \\
4(12) \\
2(6) \\
4(12)\end{array}$ \\
\hline
\end{tabular}

${ }^{a}$ Defined as $3+$ by immunohistochemistry, and/or with FISH confirmation; one patient's diagnosis preceded routine HER2 analysis of tumors.

Table 2. Pathologic stage distribution. ${ }^{\text {a }}$

\begin{tabular}{|c|c|c|c|c|c|c|}
\hline & \multicolumn{6}{|c|}{ N-Stage } \\
\hline \multirow{5}{*}{ 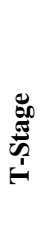 } & & pN0 & pN1 & pN2 & pN3 & $\mathrm{pNx}$ \\
\hline & pT1 & $2^{\mathrm{b}}$ & $2^{\mathrm{b}}$ & $1^{\mathrm{c}}$ & & \\
\hline & pT2 & 3 & 6 & 3 & 1 & \\
\hline & pT3 & 3 & $5^{\mathrm{b}, \mathrm{b}}$ & 3 & $3^{\mathrm{b}}$ & 1 \\
\hline & pT4 & & & & 1 & \\
\hline
\end{tabular}

${ }^{\mathrm{a}}$ As per AJCC Cancer Staging Manual, version 7; includes 5 patients who received ${ }^{\mathrm{b}}$ pre-operative chemotherapy and 1 patient who received ${ }^{\mathrm{c}}$ preoperative anastrazole.

mastectomy [14]. Thus, optimization of local control following mastectomy is critical. Standard criteria for PMRT include tumor size $\geq 5 \mathrm{~cm}$, invasion of the pectoralis and/or skin, inflammatory breast cancer, and/or lymph node involvement, as per Phase III randomized trials in Denmark [2,3] and Canada [4]. The use of PMRT in these trials confirmed by LRR benefit as well as improved OS; however, surgical margin status was not reported, and thus the benefit in this setting has not yet been evaluated prospectively.
Table 3. Treatment characteristics.

\begin{tabular}{|c|c|c|}
\hline Variable & & N (\%) \\
\hline \multicolumn{3}{|l|}{ Chemotherapy } \\
\hline None & & $6(18)$ \\
\hline Neoadjuvant & & $5(15)$ \\
\hline Adjuvant & & $23(68)$ \\
\hline Hormone Therapy & & $23(68)$ \\
\hline \multicolumn{3}{|l|}{ Radiotherapy } \\
\hline \multicolumn{3}{|l|}{$\begin{array}{l}\text { Median Duration } \\
\text { (Range) }\end{array}$} \\
\hline \multicolumn{3}{|l|}{ Chestwall Dose } \\
\hline 5000 cGy/25 & & $25(74)$ \\
\hline 5040 cGy/28 & & $9(26)$ \\
\hline Boost Dose & 48.5 days & \\
\hline 900 cGy/5 & $(41-83)$ & $10(29)$ \\
\hline 1000 cGy/5 & & $4(12)$ \\
\hline 1080 cGy/6 & & $5(15)$ \\
\hline 1260 cGy/8 & & $2(6)$ \\
\hline 1400 cGy/7 & & $1(3)$ \\
\hline 1600 cGy/8 & & $10(29)$ \\
\hline 1620 cGy/9 & & $2(6)$ \\
\hline
\end{tabular}

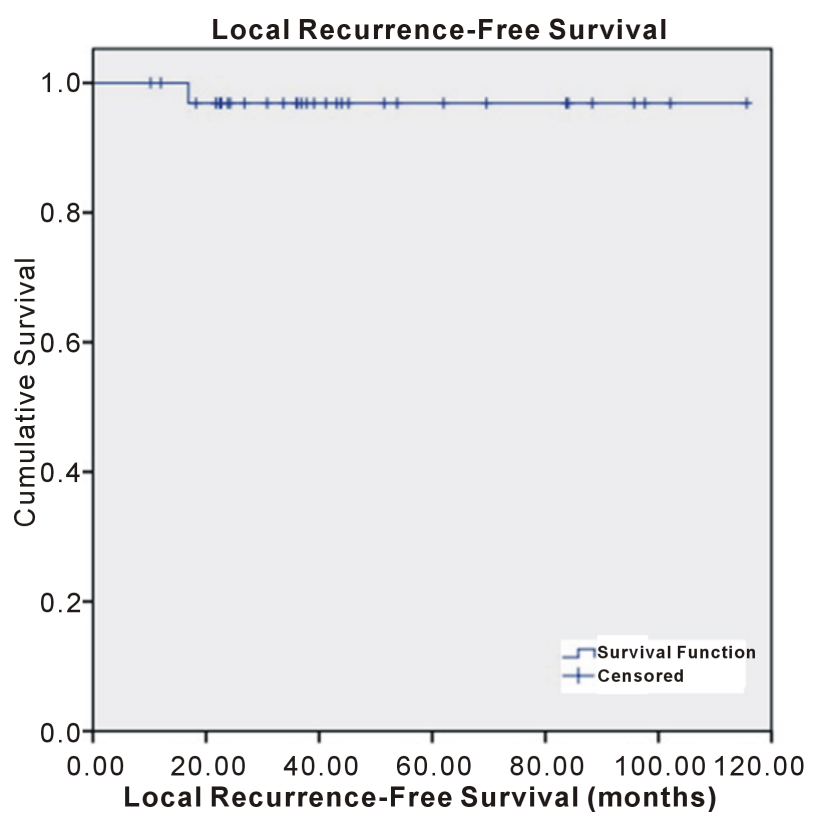

Figure 1. Freedom from locoregional failure.

Close or involved surgical margins have been associated with increased risk of LRR, particularly in the setting of additional high-risk features [5-8]. Jagsi and colleages at Massachusetts General Hospital described a series of 64 node-negative, unirradiated patients with close $(\leq 2 \mathrm{~mm})$ or involved margins at mastectomy whose 10 -year LRR was $21 \%$, as compared with $5 \%$ for 662 patients with margins $>2 \mathrm{~mm}(\mathrm{p}<0.001)$ [5]. Investigators at the Fox Chase Cancer Center similarly described elevated LRR, employing the same close/involved margin definition (estimated 8-year LRR 24\% versus 7\%), though this population included patients with 1 - 3 lymph 
nodes involved [6]. Thus, margin of $\leq 2 \mathrm{~mm}$ appears to confer a high risk of LRR, and a good population for study for the benefit and optimal dosing of PMRT.

With respect to PMRT and boost doses, retrospective evidence suggests LRR benefit for general populations of high risk patients. Feigenberg et al. of the University of Florida described detailed outcomes for PMRT in a general population of 323 patients treated with electrons to the chestwall [12]. A subset of 49 patients with involved margins were described, and at a median follow-up of 8 years, the LRR for patients with and without chestwall boost were $2 \%(1 / 41)$ and $25 \%(2 / 8)$, respectively. Similarly, investigators from the University of Miami performed a retrospective analysis of 582 patients who received PMRT, and evaluated LRR by dose [13]. As standard chestwall doses are 4500 - 5040 cGy, their investigation evaluated the relationship of total radiotherapy dose $\leq>>5040 \mathrm{cGy}$. At a median follow-up of 45 months, the estimated 5-year LRR was $5.7 \%$ versus $12.7 \%$ in patients who did or did not complete dose >5040 cGy, with benefit extending into FFF and OS as well. Additionally, the boost population had superior outcomes in several high-risk subset populations, including stage III-IVC (inflammatory) disease and triple negative receptor (hormone-insensitive, HER2-negative) status. Our findings agree with these, with similar low rate of LRR in the close/involved margin population, suggesting an advantage of RT boost overall as well in the context of established high-risk features.

Within the present study population, all patients had invasive disease, though one had only DCIS at the surgical margin. The role of radiotherapy in this setting is uncertain; following mastectomy for DCIS, the risk of LRR is minimal, though certain factors such as multi-quadrant involvement, close/involved margins, highgrade DCIS, and/or younger age may increase this risk [15-17]. Several investigations have described disease control for patients treated with mastectomy for DCIS alone, with close or involved margins. Rashtian et al. described a retrospective series of 80 patients who had undergone mastectomy for DCIS [15]. In comparing subsets by margin width, LRR developed in 5/31 (16\%) with margin $\leq 2 \mathrm{~mm}$ as compared with $1 / 49$ with margin $2.1-10 \mathrm{~mm}$. In addition, all LRR occurred in patients aged $<60$ years, a finding consistent with other investigations [16]. Thus, PMRT would be expected to confer benefit in this setting, as demonstrated by Eulau and colleagues at the Swedish Cancer Institute in Seattle, who described $100 \%$ LRR at 5 years for 15 patients who underwent PRMT for DCIS within $2 \mathrm{~mm}$ of the mastectomy margin [16]. However, not all studies have determined that close margins are sufficiently high-risk to warrant PMRT. Investigators at the University of Cali-
fornia-San Francisco described a heterogeneous population of 59 patients with surgical margin $\leq 5 \mathrm{~mm}$, of whom only one had. They concluded that PMRT in women with DCIS and close margins is unnecessary, though recommending that further investigation is required in the setting of involved margins [17].

Within the present investigation, all patients with close/involved margins received PMRT plus boost, with favorable early LRR. Going forward, we hope to compare our study population with a matched group of patients who received PMRT without boost, so as to determine whether the supplemental dose is justified in this setting. Additional follow-up will be important so as to ensure that this benefit is preserved over time.

\section{Conclusion}

This study demonstrates favorable local control with PMRT plus boost in the setting of close or involved margins after MRM. Further investigation is warranted in order to determine whether these benefits are superior to those of PMRT without boost.

\section{Acknowledgements}

All three lead authors are presently $4^{\text {th }}$ year medical students at the University of North Dakota School of Medicine and Health Sciences, and contributed equally to the design, data extraction, and manuscript development process of this study.

\section{REFERENCES}

[1] R. Zellars, "Post-Mastectomy Radiotherapy," Clinical Advances in Hematology and Oncology, Vol. 7, 2009, pp. 533-543.

[2] M. Overgaard, P. S. Hensen, J. Overgaard, et al., "Postoperative Radiotherapy in High-Risk Premenopausal Women with Breast Cancer Who Receive Adjuvant Chemotherapy," The New England Journal of Medicine, Vol. 337, 1997, pp. 949-955. http://dx.doi.org/10.1056/NEJM199710023371401

[3] M. Overgaard, M. B. Jensen, J. Overgaard, et al., "Postoperative Radiotherapy in High-Risk Postmenopausal Breast-Cancer Patients Given Adjuvant Tamoxifen: Danish Breast Cancer Cooperative Group Dbcg 82c Randomised Trial," Lancet, Vol. 353, No. 9165, 1999, pp. 1641-1648.

http://dx.doi.org/10.1016/S0140-6736(98)09201-0

[4] J. Ragaz, S. M. Jackson, N. Le, et al., “Adjuvant Radiotherapy and Chemotherapy in Node-Positive Premenopausal Women with Breast Cancer," The New England Journal of Medicine, Vol. 337, 1997, pp. 956-962. http://dx.doi.org/10.1056/NEJM199710023371402

[5] R. Jagsi, R. A. Raad, S. Goldberg, et al., "Locoregional Recurrence Rates and Prognostic Factors for Failure in 
Node-Negative Patients Treated with Mastectomy: Implications for Postmastectomy Radiation," International Journal of Radiation Oncology * Biology * Physics, Vol. 62, No. 4, 2005, pp. 1035-1039.

http://dx.doi.org/10.1016/j.ijrobp.2004.12.014

[6] G. M. Freedman, B. L. Fowble, A. L. Hanlon, et al., “A Close or Positive Margin Is Not an Indication for Chest Wall Irradiation Except in Women Aged Fifty or Younger," International Journal of Radiation Oncology * Biology * Physics, Vol. 41, No. 3, 1998, pp. 599-605. http://dx.doi.org/10.1016/S0360-3016(98)00103-5

[7] P. T. Truong, I. A. Olivotto, C. H. Speers, et al., “A Positive Margin Is Not Always an Indication for Radiotherapy after Mastectomy in Early Breast Cancer," International Journal of Radiation Oncology * Biology * Physics, Vol. 58, No. 3, 2004, pp. 797-804. http://dx.doi.org/10.1016/S0360-3016(03)01626-2

[8] P. T. Truong, M. Lesperance, A. Culhaci, et al., "Patient Subsets with T1-T2, Node-Negative Breast Cancer at High Locoregional Recurrence Risk after Mastectomy,” International Journal of Radiation Oncology * Biology * Physics, Vol. 62, No. 1, 2005, pp. 175-182. http://dx.doi.org/10.1016/j.ijrobp.2004.09.013

[9] H. Bartelink, J. C. Horiot, P. Poortmans, et al., "Recurrence Rates after Treatment of Breast Cancer with Standard Radiotherapy with or without Additional Radiation," The New England Journal of Medicine, Vol. 345, 2001, pp. 1378-1387. http://dx.doi.org/10.1056/NEJMoa010874

[10] H. Bartelink, J. C. Horiot, P. M. Poortmans, et al., "Impact of Higher Radiation Dose on Local Control and Survival in Breast-Conserving Therapy of Early Breast Cancer: 10-Year Results of the Randomized Boost versus No Boost EORTC 22881-10882 Trial,” Journal of Clinical Oncology, Vol. 25, No. 22, 2007, pp. 3259-3265. http://dx.doi.org/10.1200/JCO.2007.11.4991

[11] P. Romestaing, Y. Lehingue, C. Carrie, et al., "Role of a 10-Gy Boost in the Conservative Treatment of Early Breast Cancer: Results of a Randomized Clinical Trial in
Lyon, France,” Journal of Clinical Oncology, Vol. 15, 1997, pp. 963-968.

[12] S. J. Feigenberg, N. P. Mendenhall, R. K. Benda and C. G. Morris, "Postmastectomy Radiotherapy: Patterns of Recurrence and Long-Term Disease Control Using Electrons," International Journal of Radiation Oncology * Biology * Physics, Vol. 56, No. 3, 2003, pp. 716-725. http://dx.doi.org/10.1016/S0360-3016(03)00112-3

[13] J. E. Panoff, C. Takita, J. Hurley, et al., "Higher Chest Wall Dose Results in imProved Locoregional Outcome in Patients Receiving Postmastectomy Radiation,” International Journal of Radiation Oncology * Biology * Physics, Vol. 82, No. 3, 2012, pp. 1192-1199. http://dx.doi.org/10.1016/j.ijrobp.2011.02.045

[14] A. Chagpar, K. M. Kuerer, K. K. Hunt, E. A. Strom and T. A. Buchholz, "Outcome of Treatment for Breast Cancer Patients with Chestwall Recurrence According to Initial Stage: Implications for Post-Mastectomy Radiation Therapy," International Journal of Radiation Oncology * Biology * Physics, Vol. 57, No. 1, 2003, pp. 128-135. http://dx.doi.org/10.1016/S0360-3016(03)00431-0

[15] A. Rashtian, S. Iganej, I. A. Liu, et al., "Close or Positive Margins after Mastectomy for DCIS: Pattern of Relapse and Potential Indications for Radiotherapy,” International Journal of Radiation Oncology * Biology * Physics, Vol. 72, No. 4, 2008, pp. 1016-1020. http://dx.doi.org/10.1016/j.ijrobp.2008.06.1954

[16] S. M. Eulau and J. D. Beatty, "The Role of Adjuvant Radiotherapy after Mastectomy in Ductal Carcinoma in Situ, Breast," International Journal of Radiation Oncology * Biology * Physics, Suppl.69, 2007

[17] L. W. Chan, J. Rabban, S. Hwang, et al., "Is Radiation Indicated in Patients with Ductal Carcinoma in Situ and Close or Positive Mastectomy Margins?” International Journal of Radiation Oncology * Biology * Physics, Vol. 80, No. 1, 2011, pp. 25-30. http://dx.doi.org/10.1016/j.ijrobp.2008.06.1954 